\title{
Charged Particle's Tunneling in a Modified Reissner-Nordstrom Black Hole
}

\author{
Cheng-Zhou Liu
}

Received: 16 April 2013 / Accepted: 7 August 2013 / Published online: 29 August 2013

(C) The Author(s) 2013. This article is published with open access at Springerlink.com

\begin{abstract}
In the tunneling framework of Hawking radiation, charged particle's tunneling in the modified Reissner-Nordstrom black hole from gravity's rainbow is investigated. To this end, following the Schwarzschild solution in gravity's rainbow, the metric of the modified Reissner-Nordstrom black hole is given. In the tunneling process, the metric fluctuation is taken into account, due to not only the energy conservation and electric charge conservation, but also the spacetime quantum effects. The calculation shows out that the emission rate satisfies the first law of black hole thermodynamics and is consistent with an underlying unitary theory. In addition, it is found that the entropy of the modified black hole is different to the Benkestein-Hawking entropy and the quantum corrections of the entropy appears.
\end{abstract}

Keywords Quantum tunneling · Modified black hole · Quantum effect

\section{Introduction}

Hawking radiation is a central question concerning the quantum physics of black holes [1, 2]. In the tunneling framework [3-5] (for a review see [6]), Hawking radiation is presented semi-classically as a tunneling process of outgoing particles from the event horizon. The crucial point of the tunneling framework is that the self-gravitation of the tunneling particles is taken into account and hence the background is allowed to fluctuate. By calculating the imaginary part of the tunneling action, the emission rate is related to the change of black hole entropies before and after the emission. This implies that information conservation of black holes is possible. Thus, the method not only presented a description on Hawking radiation originating from the tunneling effect of particle near the event horizon [1,2], but also give a way for a possible resolution of the information loss paradox [7, 8].

There are two methods for calculating the tunneling action, that is, the radial trajectory method [4-6] and the Hamilton-Jacobi method [3, 6]. Using the radial trajectory method, the tunneling research has been done in many cases including different black holes [9-12],

C.-Z. Liu ( $\square)$

Department of Physics, Shaoxing University, Shaoxing 312000, China

e-mail: czlzj20@aliyun.com 
different emitted particles [10-14] and cosmological horizons [13, 15, 16]. Also, using the Hamilton-Jacobi method, the tunneling analysis has been carried out and expanded, for example in Rindler spacetime [3,17-22]. In these tunneling researches, the result that the tunneling probability is related to the change of Benkestein-Hawking (B-H) entropies before and after the emission are obtained. But, it is well known that, the quantum effects of spacetime should be considered and the B-H entropy can be endowed with quantum corrections [23-25]. Thence, by the Hamilton-Jacobi method, the quantum correction to the tunneling program was discussed and the quantum corrected entropy of black holes was calculated [26-33]. Also, in the radial trajectory method, the quantum effects of spacetime was considered and the emission rate was related the quantum corrected entropy of black hole [34-43]. These results show that, considering the quantum corrections of black hole entropy and spacetime, information conservation of black hole is still possible.

Further, some resent progress and insights for the tunneling program have been proposed [19-22, 44-54]. Therein, with the aid of density matrix techniques, the tunneling mechanism was reformulated to directly find the radiation spectrum [44, 45] and the approach was applied in different gravity to obtain the entropy spectrum of black holes [46]. It is noteworthy that, by this approach, the black body spectrum was obtained [21, 22, 44, 45]. In addition, some proposals on the calculation of particle's action and the acquisition of black hole information have been proposed [47-54]. But, in the literatures, much less attention was paid to the case of charged particle's tunneling in the quantum corrected spacetime [42].

Recently, a Planck scale modified special relativity namely doubly special relativity (DSR) has been put forward (for a review see [55]). In the context of DSR, the deformed spacetimes geometry has been investigated and some proposals have been put forward [56-61]. Therein, a spacetime geometry named as gravity's rainbow has been presented $[56,57]$. The main feature of the deformed spacetime is that the geometry depend on the energies of particles moving in it. The energy dependence can be seen as the quantum effects of the deformed spacetime. The modified Schwarzschild solution in gravity's rainbow has been given [57]. Further, the thermodynamics [39, 40, 62-64] and geometry structure [65-67] of the modified Schwarzschild black hole have been investigated.

In this paper, charged particle's tunneling in the modified Reissner-Nordstrom (R-N) black hole from gravity's rainbow is investigated. Our main aim is to investigate the influence of the spacetime quantum effect to the tunneling of charged particles. On the other hand, we also hope to give a look over on the modified black hole. For this, following [57], we firstly write the metric of the modified R-N black hole from gravity's rainbow. Further, some thermodynamic quantities of the modified black hole are given. Next, using the radial trajectory method of tunneling framework, the emission rate of charged particle in the modified R-N spacetime is calculated. Here, while charged particles tunnel though the event horizon, the metric fluctuation is taken into account, due to not only the energy conservation and electric charge conservation but also the quantum effects of the spacetime. The calculation shows out that, the emission rate satisfies the first law of black hole thermodynamics and is consistent with an underlying unitary theory. In addition, it is found that the entropy of the modified black hole is different to B-H entropy and the quantum corrections of the entropy is discussed.

The paper is organized as follows. In Sect. 2, the modified R-N solution from gravity's rainbow is given. Then in Sect. 3, using the radial trajectories method of tunneling framework, the emission rate of charged particle from the modified R-N black hole is calculated. Also, the entropy with quantum corrections for the modified black hole is investigated. The last part is the summary and discussion. 


\section{The Modified R-N Black Holes from Gravity's Rainbow}

DSR was proposed to preserve the relativity of inertial frames when keeping the Planck energy as a universal constant for all inertial observers [55]. It's staring point and main result is the modified dispersion relation (MDR) as

$$
E^{2} f_{1}^{2}\left(E ; E_{p}\right)-p^{2} f_{2}^{2}\left(E ; E_{p}\right)=m_{0}^{2},
$$

where $f_{1}$ and $f_{2}$ are two energy functions from which rotational symmetry can be preserved, and $E_{p}=1 / \sqrt{8 \pi}$ is the Planck energy. Here and in this paper, the unit of $c=G=\hbar=k=1$ is used.

In the context of DSR, different deformed spacetimes have been proposed [56-61]. Among them, it is posed that the flat spacetimes has the invariant [56]

$$
d s^{2}=-\frac{d t^{2}}{f_{1}^{2}}+\frac{d r^{2}}{f_{2}^{2}}+\frac{r^{2}}{f_{2}^{2}} d \Omega^{2} .
$$

Then, the geometry are described by one parameter family of metric as a function of particle energy observed by an inertial observer. That is to say, particles with different energies will probe different DSR spacetime. Thus, the DSR spacetime is endowed with an energy dependent quadratic invariant, namely rainbow metric. In the low energy realm i.e. $E / E_{p} \ll 1$, $f_{1}$ and $f_{2}$ approach to unit and the DSR spacetime can turn to the usual flat spacetime. This is consistent with the correspondence principle.

By extending DSR and rainbow metric to incorporate curvature, a one parameter family of energy-momentum tensors $T_{\mu \nu}(E)$ are defined and the Einstein equations can be modified as [57]

$$
G_{\mu \nu}(E)=8 \pi G(E) T_{\mu \nu}(E)+g_{\mu \nu}(E) \Lambda(E),
$$

where $G(E)$ is an energy dependent Newton's constant. The energy dependence of $G(E)$ implies that the effective gravitational coupling will depend on the energy of test particle and will satisfy a renormalization group equation. Using probes of energy $E=0$, the $G(E)$ are turn to the physical Newton's constant $G(0)=G=1$. Also, the $\Lambda(E)$ is defined as the energy dependent cosmological constants and is consistent with the correspondence principle. Accordingly, gravity's rainbow was presented and the modified Schwarzschild solution was given as [57]

$$
d s^{2}=-\frac{1}{f_{1}^{2}}\left(1-\frac{2 M}{r}\right) d t_{s}^{2}+\frac{1}{f_{2}^{2}}\left(1-\frac{2 M}{r}\right)^{-1} d r^{2}+\frac{r^{2}}{f_{2}^{2}} d \Omega^{2} .
$$

Now, following (4), we give the modified R-N black hole in gravity's rainbow. The general form for a spherically symmetric metric in gravity's rainbow is

$$
d s^{2}=-\frac{F^{\prime}\left(r^{\prime}\right)}{f_{1}^{2}} d t^{\prime 2}+\frac{H^{\prime}\left(r^{\prime}\right)}{f_{2}^{2}} d r^{\prime 2}+\frac{r^{\prime 2}}{f_{2}^{2}}\left(d \theta^{2}+\sin ^{2} \theta d \varphi^{2}\right),
$$

where $t^{\prime}, r^{\prime}, \theta$ and $\varphi$ are energy independent coordinates. The energy dependent area and time coordinates are, respectively,

$$
r(E)=\frac{r^{\prime}}{f_{2}}, \quad t(E)=\frac{t^{\prime}}{f_{1}} .
$$

It is seen that, the energy dependence of the metric and coordinates is expressed by the energy functions $f_{1}$ and $f_{2}$. Further, the energy dependent metric functions can be defined as

$$
F(r(E), E)=F^{\prime}\left(r^{\prime}\right), \quad H(r(E), E)=H^{\prime}\left(r^{\prime}\right)
$$


Then the metric (5) can be written as

$$
d s^{2}=-F(r(E), E) d t^{2}+H(r(E), E) d r^{2}+r^{2} d \Omega^{2} .
$$

This is a one parameter family of metrics. For each $E$, by the extended Birkoff's theorem that any spherically symmetric solution of the electromagnetic vacuum field equations must be the R-N metric, we have

$$
F(r(E), E)=H^{-1}(r(E), E)=\left(1-\frac{C(E)}{r(E)}+\frac{D(E)}{r^{2}(E)}\right),
$$

where the integration constants of $C(E)$ and $D(E)$ are now energy dependent. From (6), we can express the energy dependence of $C(E)$ and $D(E)$ in the energy functions $f_{1}$ and $f_{2}$, that is

$$
F(r(E), E)=H^{-1}(r(E), E)=\left(1-\frac{C(E) f_{2}}{r^{\prime}(E)}+\frac{D(E) f_{2}^{2}}{r^{\prime 2}(E)}\right)=F^{\prime}\left(r^{\prime}\right) .
$$

According to correspondence principle, at $E=0$, we must have $G(0)=G=1$ and

$$
C=2 M, \quad D=Q^{2} \text {. }
$$

Thus we obtain

$$
C(E)=\frac{2 M}{f_{2}}, \quad D(E)=\frac{Q^{2}}{f_{2}^{2}},
$$

where $M$ and $Q$ denote the energy independent mass parameter and electric charge parameter, respectively. Then, substituting (12) and (9) into (8) and using the energy independent coordinates, the modified R-N metric from gravity's rainbow is obtained as

$$
d s^{2}=-\frac{1}{f_{1}^{2}}\left(1-\frac{2 M}{r}+\frac{Q^{2}}{r^{2}}\right) d t_{s}^{2}+\frac{1}{f_{2}^{2}}\left(1-\frac{2 M}{r}+\frac{Q^{2}}{r^{2}}\right)^{-1} d r^{2}+\frac{r^{2}}{f_{2}^{2}} d \Omega^{2} .
$$

We see that, by way of energy functions $f_{1}$ and $f_{2}$, the modified R-N spacetime depend on the energy of probe particle. That is, if a given observer probes the spacetime using the quanta with different energies, the observer will conclude that the spacetime geometry have different effective descriptions. Then, the modified black hole is endowed with quantum effects behaved as the energy dependence.

In addition, the explicit MDR models and forms of $f_{1}$ and $f_{2}$ have been proposed [68]. Here, for convenience, we take

$$
f_{1}=f_{2}^{-1}=\sqrt{f} .
$$

Then, metric (13) can be simplified as

$$
d s^{2}=-\frac{1}{f}\left(1-\frac{2 M}{r}+\frac{Q^{2}}{r^{2}}\right) d t_{s}^{2}+f\left(1-\frac{2 M}{r}+\frac{Q^{2}}{r^{2}}\right)^{-1} d r^{2}+f r^{2} d \Omega^{2} .
$$

From the metric (15), we can see the event horizon $r_{+}$and the inner horizon $r_{-}$of the modified R-N black hole are

$$
r_{ \pm}=M \pm \sqrt{M^{2}-Q^{2}} .
$$

Obviously, they are universal for all observers and are the usual values in the usual R-N black hole. However, the area of the event horizon

$$
A=f 4 \pi r_{+}^{2}
$$

is energy dependent and is different to the area of the usual black hole. 
In addition, from (15), the surface gravity on the event horizon can be obtained as

$$
\kappa=-\frac{1}{2} \lim _{r \rightarrow r_{+}} \sqrt{\frac{-g^{r r}}{g^{t t}}} \frac{1}{g^{t t}} \frac{\partial g^{t t}}{\partial r}=\frac{\sqrt{M^{2}-Q^{2}}}{2 f\left(M+\sqrt{M^{2}-Q^{2}}\right)^{2}} .
$$

Further, for gravity's rainbow, Hawking temperature can be obtained from the surface gravity [62]. Then, considering (16), the temperature of the modified black hole can be written as

$$
T=\frac{\kappa}{2 \pi}=\frac{1}{4 \pi f} \frac{r_{+}-r_{-}}{r_{+}^{2}} .
$$

We see that, the Hawking temperature is also energy dependent. That is, using the particles with different energies, an observer at infinity will probe different effective temperatures. It can be said, the energy dependence of the temperature and area of the black hole is originated in the quantum effects of gravity's rainbow. Further, it can be expected that, the quantum effects of the spacetime should has some influence on particle's tunneling.

Besides, it is seen that, the modified R-N black hole is asymptotically DSR. Then, considering the asymptotically DSR spacetimes has equality with the usual asymptotically flat spacetimes [65], we obtain the total Arnowitt-Deser-Misner (ADM) mass $M_{A D M}$ for the modified black hole (15) as

$$
M_{A D M}=-\lim _{r \rightarrow \infty} \frac{1}{8 \pi} \int_{s} \varepsilon_{a b c d} \nabla^{c} \xi^{d}=M .
$$

Also, the ADM charge of the spacetimes can be expressed by

$$
Q_{A D M}=\frac{1}{4 \pi} \int_{s} \varepsilon_{a b c d} F^{c d}=Q .
$$

That is, the ADM mass and charge of the modified R-N black hole are the same as from the usual R-N black hole.

\section{Emission Rate of Charged Particle in the Modified R-N Black Hole}

Gravity's rainbow should be seen as a low energy effect of quantum gravity. It is that, the modified black hole of (15) can be seen as a coarse grained model of spacetime at semiclassical level. Here, for investigating Hawking radiation in the modified R-N black holes, we assume the tunneling program as a semi-classical method studying Hawking radiation can be still used in gravity's rainbow.

In the tunneling program, using the WKB approximation, the emission rate of tunneling particle can be obtained from the imaginary part of the action for the tunneling trajectory, namely [3-5]

$$
\Gamma \sim \exp (-2 \operatorname{Im} I)
$$

Further, the imaginary part of the action in Schwarzschild black hole can be derived as

$$
\operatorname{Im} I=\operatorname{Im} \int p_{r} d r
$$

It should be pointed that, there are some views and insights on the relations (22) and (23) [47-52]. It have been proposed that the action $I=\int p_{r} d r$ is not invariant under canonical transformations. The canonical invariant is $I=\oint p_{r} d r$ and the formula (22) with a 
factor of $1 / 2$ in the exponent is canonically invariant. But, using the canonical invariant, the obtained temperature was higher than the Hawking temperature by a factor of 2 and the "factor 2 problem" was presented. But, in terms of an overlooked temporal contribution to the tunneling amplitude, this problem was given a resolution. That is, when the temporal contribution was considered, the correct temperature was obtained by using the canonically invariant tunneling amplitude.

In addition, when we calculate the action of charged particle in the modified R-N black holes (15), the effect of electro-magnetic field should be taken into account. That is, we should consider the matter-gravity system consisting of the black hole and the electromagnetic field outside the black hole. For the electromagnetic field in the spacetimes, treating the black hole as a charged conducting sphere [69], the non-zero component of electro-magnetic potential is

$$
A_{t}=\frac{Q}{r}
$$

The Lagrangian function for the matter-gravity system is

$$
L=L_{m}+L_{e},
$$

where $L_{e}=-\frac{1}{4} F_{\mu \nu} F^{\mu \nu}$ is the Lagrangian function of the electromagnetic field corresponding to the generalized coordinates $A_{t}$ [70]. Considering $A_{t}$ is an ignorable coordinate, to eliminate this degree of freedom completely, the Lagrangian function of the matter-gravity system should be written as $L-P_{A_{t}} A_{t}$, in which $P_{A_{t}}$ is the canonical momentum conjugate to $A_{t}$. Thus, we can obtain the action of the matter-gravity system as

$$
I=\int_{t_{i}}^{t_{f}}\left(L-P_{A_{t}} \dot{A_{t}}\right) d t=\int_{t_{i}}^{t_{f}}\left(P_{t}+P_{r} \dot{r}-P_{A_{t}} \dot{A_{t}}\right) d t,
$$

where $p_{t}$ and $p_{r}$ and are the conjugate momentum corresponding to Painleve's coordinates $t$ and $r, t_{i}$ and $t_{f}$ the Painleve coordinate times before and after the charged particle's emission, respectively. Considering only the second and third term in (26) contribute to the imaginary part of the action, then we can obtain

$$
\begin{aligned}
\operatorname{Im} I & =\operatorname{Im} \int_{t_{i}}^{t_{f}}\left(P_{r} \dot{r}-P_{A_{t}} \dot{A}_{t}\right) d t=\operatorname{Im} \int_{r_{i}}^{r_{f}}\left(P_{r}-\frac{P_{A_{t}} \dot{A}_{t}}{\dot{r}}\right) d r \\
& =\operatorname{Im} \int_{r_{i}}^{r_{f}} \int_{(0,0)}^{\left(P_{r}, P_{A_{t}}\right)}\left(d p_{r}^{\prime}-\frac{\dot{A}_{t}}{\dot{r}} d P_{A_{t}}^{\prime}\right) d r,
\end{aligned}
$$

where $r_{i}$ and $r_{f}$ are the locations of the horizons corresponding $t_{i}$ and $t_{f}$, respectively.

To proceed with an explicit computation on (27), the coordinate singularity at the horizon must be removed. For the modified R-N black holes, following [72], we carry on a Painleve type coordinate transformation. That is, letting

$$
d t_{s}=d t-F(r) d r
$$

and

$$
f\left(1-\frac{2 M}{r}+\frac{Q^{2}}{r^{2}}\right)^{-1}-\frac{1}{f}\left(1-\frac{2 M}{r}+\frac{Q^{2}}{r^{2}}\right) F^{2}(r)=1,
$$


we have

$$
\begin{aligned}
d s^{2}= & -\frac{1}{f}\left(1-\frac{2 M}{r}+\frac{Q^{2}}{r^{2}}\right) d t^{2}+2 \sqrt{1-\frac{1}{f}\left(1-\frac{2 M}{r}+\frac{Q^{2}}{r^{2}}\right)} d t d r+d r^{2} \\
& +f r^{2}\left(d \theta^{2}+\sin ^{2} \theta d \varphi^{2}\right) .
\end{aligned}
$$

It is easy to find that, to implement the calculation on the emission rate of particle tunneling through the event horizon, the Painleve-like metric of the Planck scale corrected spacetime has a number of attractive features. Firstly, as expected, none of the components of either the metric or the inverse metric diverges at the horizon. Secondly, the coordinate system has Killing vector $\partial / \partial t$. Thirdly, in the new form of the line element, constant time slices are just flat Euclidean in radial. Fourthly, the components of the new metric satisfy Landau's condition of the coordinate clock synchronization [73], namely

$$
\frac{\partial}{\partial x^{i}}\left(\frac{g_{0 j}}{g_{00}}\right)=\frac{\partial}{\partial x^{j}}\left(\frac{g_{0 i}}{g_{00}}\right) \quad(i, j=1,2,3) .
$$

Finally, the metric has the Planck scale effects showed as the energy dependence. This denotes that, even if the black hole has a fixed mass parameter, the emitted particles with different energies will be affected by different metric.

On other hand, if we enforce energy conservation and charge conservation of the spacetimes, when the particle tunnels out the horizon, the mass and charge of the black hole should vary. That is, the self-gravitation of emitted particle should affect the background geometry. In spherical symmetry spacetime, the self-gravitation effects of emitted shell have been investigated in detail [69]. Here, we assume the tunneling particle has energy $\omega$ and electric charge $q$ measured at infinity. Then, considering (20) and (21), when the tunneling particle treated as a spherical shell radiate outside the event horizon, the parameters $M$ and $Q$ in the black hole (30) should be replaced with $M-\omega$ and $Q-q$, respectively [3-5, 69]. In fact, this is consistent with Birkhoff's theorem. The theorem tell us that, in the spherical symmetry spacetime, the only effect on the geometry due to the s-wave is to provide a junction condition for matching the total mass inside and outside the shell. Then we get the geometry between the horizon and the spherical shell as

$$
\begin{aligned}
d s^{2}= & -\frac{1}{f}\left(1-\frac{2(M-\omega)}{r}+\frac{(Q-q)^{2}}{r^{2}}\right) d t^{2} \\
& +2 \sqrt{1-\frac{1}{f}\left(1-\frac{2(M-\omega)}{r}+\frac{(Q-q)^{2}}{r^{2}}\right)} d t d r \\
& +d r^{2}+f r^{2}\left(d \theta^{2}+\sin ^{2} \theta d \varphi^{2}\right) .
\end{aligned}
$$

Thus, we see that, the event horizons before and after the particle's emission are $r_{i}=$ $r_{+}(M, Q)=M+\sqrt{M^{2}-Q^{2}}$ and $r_{f}=r_{+}(M-\omega, Q-q)=(M-\omega)+$ $\sqrt{(M-\omega)^{2}-(Q-q)^{2}}$, respectively. That is, by the shrinking of the black hole, the tunneling barrier is created by the emitted particle itself due to the energy conservation and charge conservation of the spacetime. Moreover, the barrier location is not affected by the Planck scale effects of the black hole-emitted particle system.

Next, we apply the Hamilton's equation

$$
\dot{r}=\left(\frac{d H}{d p_{r}}\right)_{\left(r ; A_{t}, P_{A_{t}}\right)}=\frac{d M_{A D M}^{\prime}}{d p_{r}}, \quad \dot{A}_{t}=\left(\frac{d H}{d p_{A_{t}}}\right)_{\left(A_{t} ; r, p_{r}\right)}=\frac{d E_{Q}^{\prime}}{d p_{A_{t}}} .
$$


Here $M_{A D M}^{\prime}=M^{\prime}=\left(M-\omega^{\prime}\right)$ is the mass of the modified black hole after emitting a particle with energy $E^{\prime}=\omega^{\prime}, E_{Q}^{\prime}$ represents the energy of the electro-magnetic field and

$$
d E_{Q}^{\prime}=\frac{Q^{\prime}}{r_{+}^{\prime}} d Q^{\prime}
$$

$Q^{\prime}=Q-q^{\prime}$ is the electric charge of the black hole after emitting electric charge $q^{\prime}$. Then, substituting (33) and (34) into (27) and switching the integral order, the imaginary part of the action reads

$$
\begin{aligned}
\operatorname{Im} I & =\operatorname{Im} \int_{r_{i}}^{r_{f}} \int_{(M, Q)}^{(M-\omega, Q-q)} \frac{d M^{\prime}-d E_{Q}^{\prime}}{\dot{r}} d r \\
& =\operatorname{Im} \int_{(M, Q)}^{(M-\omega, Q-q)} \int_{r_{i}}^{r_{f}}\left(d M^{\prime}-\frac{Q^{\prime}}{r_{+}^{\prime}} d Q^{\prime}\right) \frac{d r}{\dot{r}} .
\end{aligned}
$$

In addition, in term of quantum mechanics, tunneling across potential barrier is an instantaneous process. That is, the particle tunneling into and out the barrier are two simultaneous events. Then, according to Landau's condition of the coordinate clock synchronization, the coordinate time difference of these two events is

$$
\Delta t=-\frac{g_{t r}}{g_{t t}} \Delta r
$$

where $\Delta r$ is the potential barrier width. Therefore, the radial trajectory of the outgoing charged particle with energy $\omega^{\prime}$ and electric charge $q^{\prime}$ can be obtained as

$$
\begin{aligned}
\dot{r} & =\frac{d r}{d t}=-\frac{1}{2} \frac{g_{t t}\left(M^{\prime}, Q^{\prime}\right)}{g_{t r}\left(M^{\prime}, Q^{\prime}\right)}=\frac{1}{2 f} \frac{\left(1-\frac{2 M^{\prime}}{r}+\frac{Q^{\prime 2}}{r^{2}}\right)}{\sqrt{1-\frac{1}{f}\left(1-\frac{2 M^{\prime}}{r}+\frac{Q^{\prime 2}}{r^{2}}\right)}} \\
& =\frac{1}{2 f} \frac{\left(r-r_{+}^{\prime}\right)\left(r-r_{-}^{\prime}\right)}{r \sqrt{r^{2}-\frac{1}{f}\left(r-r_{+}^{\prime}\right)\left(r-r_{-}^{\prime}\right)}},
\end{aligned}
$$

where $r_{+}^{\prime}=M^{\prime}+\sqrt{\left(M^{\prime}\right)^{2}-\left(Q^{\prime}\right)^{2}}$ is the location of horizon after the emission.

Substituting (37) into (35), the imaginary part of the action can be obtained as

$$
\operatorname{Im} I=\operatorname{Im} \int_{(M, Q)}^{(M-\omega, Q-q)} \int_{r_{i}}^{r_{f}}\left(d M^{\prime}-\frac{Q^{\prime}}{r_{+}^{\prime}} d Q^{\prime}\right) 2 f \frac{r \sqrt{r^{2}-f^{2}\left(r-r_{+}^{\prime}\right)\left(r-r_{-}^{\prime}\right)}}{\left(r-r_{+}^{\prime}\right)\left(r-r_{-}^{\prime}\right)} d r .
$$

Considering the particle tunneling through the horizon, it is seen that $r_{+}^{\prime}$ is a single pole in (38). Then the integral can be evaluated by deforming the contour around the pole. In this way, we finished the integral over $r$ and get

$$
\operatorname{Im} I=-2 \pi \int_{(M, Q)}^{(M-\omega, Q-q)} f \frac{r_{+}^{\prime 2}}{r_{+}^{\prime}-r_{-}^{\prime}}\left(d M^{\prime}-\frac{Q^{\prime}}{r_{+}^{\prime}} d Q^{\prime}\right) .
$$

Now, for the modified black hole in the tunneling process, we apply the first law of black hole thermodynamics

$$
d M^{\prime}-\frac{Q^{\prime}}{r_{+}^{\prime}} d Q^{\prime}=T^{\prime} d S^{\prime}
$$

Then, substituting (40) into (39) and considering the temperature formula (19), we have

$$
2 \pi f \frac{r_{+}^{\prime 2}}{r_{+}^{\prime}-r_{-}^{\prime}}\left(d M^{\prime}-\frac{Q^{\prime}}{r_{+}^{\prime}} d Q^{\prime}\right)=\frac{1}{2} d S^{\prime}
$$


and

$$
\operatorname{Im} I=-\frac{1}{2} \int_{S}^{S+\Delta S} d S^{\prime}=-\frac{1}{2} \Delta S
$$

where $\Delta S=S(M-\omega, Q-q)-S(M, Q)$ is the difference of the black hole entropies for the modified black hole before and after the emission.

Substituting (42) into (22), the tunneling probability of charged particles in the gravity's rainbow is obtained as

$$
\Gamma=\exp (-2 \operatorname{Im} I)=\exp (\Delta S)=\exp [S(M-\omega, Q-q)-S(M, Q)] .
$$

It is seen that, the emission rate is related to the change of the entropies of the modified black hole. This is consistent with an underlying unitary theory. In addition, the result is identical to the previous results in the literatures [3-6, 9-13, 15-43]. Further, on the tunneling result, a rigorous proof has been given [33]. But, for the modified black hole, the entropy and the obtained emission rate should include quantum corrections arising from the quantum effects of the spacetime.

From (41), the differential entropy for the modified black holes can be obtained as

$$
d S=4 \pi f \frac{r_{+}^{2}}{r_{+}-r_{-}}\left(d M-\frac{Q}{r_{+}} d Q\right)=4 \pi f \frac{r_{+}}{r_{+}-r_{-}}\left(r_{+} d M-Q d Q\right) .
$$

By means of the identity

$$
r_{+} d M-Q d Q=\frac{1}{2}\left(r_{+}-r_{-}\right) d r_{+}
$$

and considering (17), we have

$$
d S=2 \pi f d r_{+}^{2}=\frac{d A}{4}-\frac{A}{4} \frac{d f}{f} .
$$

It is seen that, the B-H entropy $S_{B H}=\frac{A}{4}$ of the black hole can be derived from the first part of (46). The second part is particle energy dependent and should be seen as the quantum correction $S_{Q C}$ to the B-H entropy. Then, the entropy of the modified black hole is written as

$$
S=S_{B H}+S_{Q C}=\frac{A}{4}+\int \frac{A}{4} \frac{d f}{f} .
$$

This is a different result with the case in the usual R-N black hole, in which $S=\frac{A}{4}$ appear in the emission rate of charged particles [10]. Then, from (43) and (47), it is implied that, information conservation of black hole is still possible when the quantum corrections of black hole entropy are considered. In addition, the corrected item $S_{Q C}$ of the black hole entropy can be calculated by giving the specific form of the energy function $f[39,40,62,68]$. Then, from (43), the radiation spectrum with quantum corrections and the information deposited by the spectrum can be investigated further.

\section{Discussion and Summary}

In tunneling framework of Hawking radiation [3-5], charged particle's tunneling in the modified R-N black hole from gravity's rainbow is investigated. Firstly, following the Schwarzschild solution in gravity's rainbow [57], the modified R-N black hole from gravity's rainbow is given. Also, the quantum effects of the modified spacetime is discussed 
and the thermodynamic quantities of the modified black hole are calculated. Then, using the radial trajectory method $[4,5]$ of the tunneling framework, the tunneling probability of charged particle in the modified black hole is calculated. In the present tunneling, metric fluctuations are taken into account, due to not only energy conservation and electric charge conservation, but also the spacetime quantum effects. Also, the first law of black hole thermodynamics is applied. The emission rate of charged particle is related to the change of black hole entropies before and after the emission. The result is consistent with an underlying unitary theory. Due to the Planck scale effects, the entropy of the modified R-N black hole is different to the B-H entropy and the quantum corrections to the B-H entropy is discussed. The investigation implies that, considering quantum corrections of black hole entropy and spacetime, information conservation of black holes is still possible. In supporting the tunneling program, the research can provide a evidence via the charged particle's tunneling in the quantum corrected spacetime.

Acknowledgements The work was supported by the National Natural Science Foundation of China (Grant Nos. 11045005 and 11247306) and the Natural Science Foundation of Zhejiang Province of China (Grant Nos. Y6090739 and LQ13A050002). I would like to thank one of the anonymous referees for helpful comments and suggestions.

Open Access This article is distributed under the terms of the Creative Commons Attribution License which permits any use, distribution, and reproduction in any medium, provided the original author(s) and the source are credited.

\section{References}

1. Hawking, S.W.: Particle creation by black holes. Commun. Math. Phys. 43, 199 (1975)

2. Gibbons, G.W., Hawking, S.W.: Cosmological event horizons, thermodynamics and particle creation. Phys. Rev. D 15, 2752 (1977)

3. Srinivasan, K., Padmanabhan, T.: Particle production and complex path analysis. Phys. Rev. D 60, 024007 (1999). arXiv:gr-qc/9812028

4. Parikh, M.K., Wilczek, F.: Hawking radiation as tunneling. Phys. Rev. Lett. 85, 5042 (2000). arXiv: hep-th/9907001

5. Parikh, M.K.: A Secret Tunnel through the horizon. Int. J. Mod. Phys. D 13, 2355 (2004). arXiv: hep-th/0405160

6. Vanzo, L., Acquaviva, G., Criscienzo, R.D.: Tunneling methods and Hawking's radiation: achievements and prospects. arXiv:1106.4153

7. Page, D.N.: Average entropy of a subsystem. Phys. Rev. Lett. 71, 3743 (1993). arXiv:gr-qc/9305007

8. Russo, J.G.: The information problem in black hole evaporation: old and recent results. arXiv:hep-th/ 0501132

9. Vagenas, E.C.: Generalization of the KKW analysis for black hole radiation. Phys. Lett. B 559, 65 (2003). arXiv:hep-th/0209185

10. Zhang, J., Zhao, Z.: Hawking radiation of charged particles via tunneling from the Reissner-Nordstrom black hole. J. High Energy Phys. 0510, 055 (2006)

11. Jiang, Q.Q., Wu, S.Q., Cai, X.: Hawking radiation from the Kerr and Kerr-Newman black holes. Phys. Rev. D 73, 064003 (2006). arXiv:hep-th/0512351

12. Liu, C.Z., Zhang, J.Y., Zhao, Z.: Charged particle's tunneling from a dilation black hole. Phys. Lett. B 639, 670 (2006)

13. Zhang, J.Y., Zhao, Z.: Massive particle's tunneling and de Sitter tunneling. Nucl. Phys. B 725, 173 (2005)

14. Liu, C.Z., Zhao, Z.: Hawking radiation via tunneling from the general static black holes. Mod. Phys. Lett. A 23, 539 (2008)

15. Hemming, S., Keski-Vakkuri, E.: Hawking radiation from AdS black holes. Phys. Rev. D 64, 044006 (2001). arXiv:gr-qc/0005115

16. Medved, A.J.M.: Radiation via tunneling from a de Sitter cosmological horizon. Phys. Rev. D 66, 124009 (2002). arXiv:hep-th/0207247 
17. Kerner, R., Mann, R.B.: Tunneling, temperature and Taub-NUT black holes. Phys. Rev. D 73, 104010 (2006). arXiv:gr-qc/0603019

18. Akhmedova, V., Pilling, T., de Gill, A., Singleton, D.: Comments on anomaly versus WKB/tunneling methods for calculating Unruh radiation. Phys. Lett. B 673, 227 (2009). arXiv:0808.3413 [hep-th]

19. Dbraj, R.: The Unruh thermal spectrum through scalar and fermion tunneling. Phys. Lett. B 681, 185 (2009). arXiv:0908.3149 [hep-th]

20. Banerjee, R., Majhi, B.R.: Hawking black body spectrum from tunneling mechanism. Phys. Lett. B 675, 243 (2009). arXiv:0903.0250 [hep-th]

21. Umetsu, K.: Hawking radiation from Kerr-Newman black hole and tunneling mechanism. arXiv: 0907.1420 [hep-th]

22. Banerjee, R., Modak, S.K.: Quantum tunneling, blackbody spectrum and non-logarithmic entropy correction for Lovelock black holes. J. High Energy Phys. 0911, 073 (2009). arXiv:0908.2346 [hep-th]

23. Strominger, A., Vafa, C.: Microscopic origin of the Bekenstein-Hawking entropy. Phys. Lett. B 379, 99 (1996). arXiv:hep-th/9601029

24. Gour, G., Medved, A.J.M.: Thermal fluctuations and black-hole entropy. Class. Quantum Gravity 20, 3307 (2003). arXiv:gr-qc/0305018

25. Kaul, R.K., Majumdar, P.: Logarithmic correction to the Bekenstein-Hawking entropy. Phys. Rev. Lett. 84, 5255 (2000). arXiv:gr-qc/0002043

26. Banerjee, R., Majhi, B.R.: Quantum tunneling and back reaction. Phys. Lett. B 662, 62 (2008). arXiv:0801.0200

27. Banerjee, R., Majhi, B.R.: Quantum tunneling beyond semiclassical approximation. J. High Energy Phys. 06, 095 (2008). arXiv:0805.2220

28. Banerjee, R., Majhi, B.R.: Quantum tunneling and trace anomaly. Phys. Lett. B 674, 218 (2009). arXiv:0808.3688

29. Banerjee, R., Modak, S.K.: Exact differential and corrected area law for stationary black holes in tunneling method. J. High Energy Phys. 05, 063 (2009). arXiv:0903.3221

30. Modak, S.K.: Corrected entropy of BTZ black hole in tunneling approach. Phys. Lett. B 671, 167 (2009). arXiv:0807.0959

31. Banerjee, R., Majhi, B.R., Roy, D.: Corrections to Unruh effect in tunneling formalism and mapping with Hawking effect. arXiv:0901.0466 [hep-th]

32. Majhi, B.R., Samanta, S.: Hawking radiation due to photon and gravition tunneling. arXiv:0901.2258 [hep-th]

33. Mirza, B., Sherkatghanad, Z.: Corrected entropy of the rotating black hole solution of the new massive gravity using the tunneling method and Cardy formula. Phys. Rev. D 83, 104001 (2011). arXiv: 1104.0390

34. Banerjee, R., Majhi, B.R., Samanta, S.: Noncommutative black hole thermodynamics. Phys. Rev. D 77, 124035 (2008). arXiv:0801.3583

35. Michele, A.: Black hole entropy, log corrections and quantum aerosphere. Phys. Lett. B 634, 536 (2006). arXiv:gr-qc/0512071

36. Medved, A.J.M., Vagenas, E.C.: On Hawking radiation as tunneling with logarithmic corrections. Mod. Phys. Lett. A 20, 1723 (2005). arXiv:gr-qc/0505015

37. Zhang, J.Y.: Black hole quantum tunnelling and black hole entropy correction. Phys. Lett. B 668, 353 (2008). arXiv:0806.2441

38. Arzano, M., Medved, A.J.M., Vagenas, E.C.: Hawking radiation as tunneling through the quantum horizon. J. High Energy Phys. 09, 037 (2005). arXiv:hep-th/0505266

39. Liu, C.Z., Zhu, J.Y.: Hawking radiation and black hole entropy in a gravity's rainbow. Gen. Relativ. Gravit. 40, 1899 (2008). arXiv:gr-qc/0705055

40. Liu, C.Z.: Hawking radiation via tunneling of massive particles from a gravity's rainbow. Mod. Phys. Lett. A 25, 3229 (2010)

41. Kourosh, N., Hamid Mehdipour, S.: Parikh-Wilczek tunneling from noncommutative higher dimensional black holes. J. High Energy Phys. 06, 095 (2009). arXiv:0902.1945

42. Hamid Mehdipour, S.: Charged particles' tunneling from a noncommutative charged black hole. Int. J. Mod. Phys. A 25, 5543 (2010). arXiv:1004.1255

43. Hamid Mehdipour, S.: Hawking radiation as tunneling from a Vaidya black hole in noncommutative gravity. Phys. Rev. D 81, 124049 (2010). arXiv:1006.5215

44. Banerjee, R., Majhi, B.R.: Hawking black body spectrum from tunneling mechanism. Phys. Lett. B 675, 243 (2009). arXiv:0903.0250

45. Banerjee, R., Majhi, B.R.: Quantum tunneling and black hole spectroscopy. Phys. Lett. B 686, 279 (2010). arXiv:0907.4271 [hep-th]

46. Majhi, B.R.: Hawking radiation and black hole spectroscopy in Horava-Lifshitz gravity. Phys. Lett. B 686, 49 (2010). arXiv:0911.3239 
47. Akhmedov, E.T., Akhmedova, V.A., Singleton, D.: Hawking temperature in the tunneling picture. Phys. Lett. B 642, 124 (2006)

48. Akhmedova, V., Pilling, T., Singleton, D.: Thermal radiation of various gravitational backgrounds. Int. J. Mod. Phys. A 22, 1705 (2007)

49. Chowdhury, B.D.: Problems with tunneling of thin shells from black holes. Pramana 70, 3 (2008). arXiv:hep-th/0605197

50. Pilling, T.: Black Hole Thermodynamics and the factor of 2 problem. Phys. Lett. B 660, 402 (2008)

51. Akhmedova, V., Pilling, T., Singleton, D.: Temporal contribution to gravitational WKB-like calculations. Phys. Lett. B 666, 269 (2008). arXiv:0804.2289

52. Akhmedova, V., Pilling, T., Singleton, D.: Subtleties in the quasi-classical calculation of Hawking radiation. Int. J. Mod. Phys. D 17, 2453 (2008)

53. Chen, Y.X., Shao, K.N.: Invariance of the Hamilton-Jacobi tunneling method for black holes and FRW model. arXiv:1007.4367

54. Singleton, D., Vagenas, E.C., Zhu, T., Ren, J.R.: Insights and possible resolution to the information loss paradox via the tunneling picture. J. High Energy Phys. 089, 1008 (2010). arXiv:1005.3778

55. Amelino-Camelia, G.: Doubly-special relativity: first results and key open problems. Int. J. Mod. Phys. D 11, 1643 (2002). arXiv:gr-qc/0210063

56. Kimberly, D., Magueijo, J., Medeiros, J.: Non-linear relativity in position space. Phys. Rev. D 70, 084007 (2004). arXiv:gr-qc/0303067

57. Magueijo, J., Smolin, L.: Gravity's rainbow. Class. Quantum Gravity 21, 1725 (2004). arXiv:gr-qc/ 0305055

58. Galan, P., Mena Marugan, G.A.: Quantum time uncertainty in a gravity's rainbow formalism. Phys. Rev. D 70, 124003 (2004). arXiv:gr-qc/0411089

59. Galan, P., Mena Marugan, G.A.: Length uncertainty in a gravity's rainbow formalism. Phys. Rev. D 72, 044019 (2005). arXiv:gr-qc/0507098

60. Mignemi, S.: Transformations of coordinates and Hamiltonian formalism in deformed special relativity. Phys. Rev. D 68, 065029 (2003). arXiv:gr-qc/0304029

61. Hinterleitner, F.: Canonical doubly special relativity theory. Phys. Rev. D 71, 025016 (2005). arXiv: gr-qc/0409087

62. Ling, Y., Li, X., Hu, B.: Thermodynamics of modified black holes from gravity's rainbow. Mod. Phys. Lett. A 22, 2749 (2007). arXiv:gr-qc/0512084

63. Galan, P., Mena Marugan, G.A.: Entropy and temperature of black holes in a gravity's rainbow. Phys. Rev. D 74, 044035 (2006). arXiv:gr-qc/0608061

64. Garattnti, G.: Modified dispersion relations and black hole entropy. Phys. Lett. B 685, 329 (2010). arXiv:0902.3927 [gr-qc]

65. Hackett, J.: Asymptotic flatness in rainbow gravity. Class. Quantum Gravity 23, 3833 (2006)

66. Girelli, F., Liberati, S., Sindoni, L.: Planck-scale modified dispersion relations and Finsler geometry. Phys. Rev. D 75, 064015 (2007). arXiv:gr-qc/0611024

67. Carlos, L., Joel, S., Jose, V.: Geodesic structure of the Schwarzschild black hole in rainbow gravity. Mod. Phys. Lett. A 24, 1443 (2009). arXiv:0808.2601

68. Heuson, C.: Quantization of deformed special relativity. arXiv:gr-qc/0606124

69. Kraus, P., Wilczek, F.: Effect of self-interaction on charged black hole radiance. Nucl. Phys. B 437, 231 (1995). arXiv:hep-th/9411219

70. Makela, J., Repo, P.: A quantum mechanical model of the Reissner-Nordstrom black hole. Phys. Rev. D 57, 4899 (1998). arXiv:gr-qc/9708029

71. Zhang, J., Zhao, Z.: Hawking radiation of charged particles via tunneling from the Reissner-Nordstrom black hole. J. High Energy Phys. 0510, 055 (2006)

72. Kraus, P., Wilcek, F.: Some applications of a simple stationary line element for the Schwarzschild geometry. Mod. Phys. Lett. A 9, 3713 (1994)

73. Landau, L.D., Lifshitz, E.M.: The Classical Theory of Field. Pergamon, London (1975) 